Usages du français et pratiques d'enseignement en Europe balkanique, centrale et orientale - Grèce,

Serbie, Bulgarie, Moldavie, Hongrie, Allemagne, Russie - XVIIle - XXe siècles

\title{
Loukia Efthymiou, La formation des francisants en Grèce : 1836-1982.
}

\author{
Nicolas Manitakis
}

\section{(2) OpenEdition Journals}

\section{Édition électronique}

URL : https://journals.openedition.org/dhfles/4254

DOI : $10.4000 /$ dhfles.4254

ISSN : 2221-4038

\section{Éditeur}

Société Internationale pour l'Histoire du Français Langue Étrangère ou Seconde

\section{Édition imprimée}

Date de publication : 1 juin 2015

Pagination : 215-218

ISSN : 0992-7654

\section{Référence électronique}

Nicolas Manitakis, «Loukia Efthymiou, La formation des francisants en Grèce : 1836-1982. »,

Documents pour l'histoire du français langue étrangère ou seconde [En ligne], 54 | 2015, mis en ligne le 19 novembre 2017, consulté le 31 mars 2023. URL : http://journals.openedition.org/dhfles/4254 ; DOI : https://doi.org/10.4000/dhfles.4254

Ce document a été généré automatiquement le 31 mars 2023

Tous droits réservés 


\title{
Loukia Efthymiou, La formation des francisants en Grèce : 1836-1982.
}

\author{
Nicolas Manitakis
}

Publibook, 2015, 430 pages. ISBN 978-2-342-03430-1

Le livre de Loukia Efthymiou, La formation des francisants en Grèce : 1836-1982, publié aux éditions Publibook en 2015, avec une préface de Michelle Perrot (initialement publié en Grèce, en 2013, par les éditions Simmetria, sans la préface de Michelle Perrot; ISBN 978-960-11-0027-2), vient enrichir et approfondir un champ de recherches historiques, celui de l'histoire de l'enseignement du français en Grèce aux $\mathrm{XIX}^{\mathrm{e}}$ et $\mathrm{xx}^{\mathrm{e}}$ siècles, qui, depuis les années 1990, connaît un certain développement. Les travaux de Fani Sdougka, Élisabeth Papageorgiou-Provata, Kondylia Choida et Marianthi-Elisabeth Mastora et, plus récemment, ceux de Despina Provata et de David Antoniou, ont défriché, en effet, un domaine d'investigation longtemps délaissé par les historiens de l'éducation. L'étude de L. Efthymiou se distingue, cependant, déjà par sa taille (316 pages de texte, sans compter une cinquantaine de pages d'annexes), mais encore plus, par l'ampleur des sources examinées, l'étendue de la période traitée, la richesse des données exploitées, l'approche multidimensionnelle proposée et, surtout, par la pertinence des questions posées et la valeur des interprétations avancées.

3 En ce sens, le livre d'Efthymiou constitue un travail pionnier, constitutif même du champ de recherche dans lequel il s'inscrit. Il s'agit, dans l'ensemble, d'un ouvrage soigné, minutieux, bien documenté, exhaustif quant à la recherche d'informations, solide en ce qui concerne son argumentation, clair en ce qui concerne la présentation des faits et des idées. À cela s'ajoute le souci apporté par l'auteure pour faire de son ouvrage à la fois une étude historique approfondie et un véritable outil de travail. En témoigne les douze annexes, la quarantaine de tableaux qui synthétisent les informations contenues dans le texte, l'index des noms de personnes, la longue liste des textes législatifs portant sur l'enseignement du français de 1836 jusqu'en 1999, et, enfin, la bibliographie étendue.

4 L'un des mérites du travail est d'avoir réorienté la recherche historique vers des domaines peu explorés jusqu'à présent. L'attention des historiens se déplace ainsi de 
l'étude des manuels et des méthodes d'enseignement du français à celle des enseignants et des enseignantes de la langue française. Pour être plus précis, l'auteure s'intéresse plus particulièrement à la formation donnée aux professeurs grecs de français. Or, elle entend aborder ce sujet en plaçant son travail à l'intersection de l'histoire de l'éducation, des femmes et du genre et des relations internationales. Cette triple approche constitue une nouveauté et s'avère très féconde. Elle permet notamment d'imbriquer différents niveaux d'analyse: le culturel et le politique, le national et l'international et la question du genre.

5 L'ouvrage se divise en trois parties : 1) « Le temps des hommes, le temps des tentatives $(1836-1924) » 2)$ «Le temps des réalisations: collaborations et concurrences (1924-1953)» et 3) «De la naissance à la majorité d'une formation universitaire de francisants: le cas de la Section d'études françaises de l'université d'Athènes (1953-1982)». Cette division correspond à la périodisation proposée par l'historienne pour marquer les tournants qu'a connu l'histoire cent-cinquantenaire de la formation au professorat de français en Grèce. Bien que le français ait occupé une place importante dans le programme d'enseignement des établissements secondaires depuis 1836, celui-ci étant la seule langue étrangère qu'y figurait, la formation des enseignants de cette spécialité n'a fait l'objet d'aucune attention de la part des pouvoirs publics grecs avant la fin du XIX ${ }^{e}$ siècle. C'est ce qui a poussé, d'ailleurs, la diplomatie française à s'intéresser à la question, soucieuse d'assurer la diffusion de la langue française, dans un contexte de rivalité culturelle et linguistique accrue entre les puissances européennes de l'époque. Les efforts engagés dans ce domaine par le ministère des Affaires étrangères de la France ne donnèrent des résultats tangibles et durables qu'entre les deux guerres avec la mise en place, à partir de 1930, d'un « Cours spécial de préparation au professorat de français »: organisé par l'Institut français d'Athènes et officiellement reconnu par l'État grec, il fut le fruit d'une collaboration francohellénique. Le contenu de la formation donnée à l'Institut s'inspirait à la fois de l'organisation des études littéraires dans les universités françaises et du programme d'enseignement des écoles normales françaises. Dans cette deuxième partie du livre, l'auteure cherche aussi à cerner le profil des diplômé/es du « Cours spécial » en tenant compte du sexe, des études effectuées et de l'activité professionnelle exercée après l'obtention du diplôme. Elle observe ainsi que le recrutement des élèves du cours et, par extension, des professeurs grecs de français s'est progressivement " surféminisé ».

6 La troisième et dernière partie de l'ouvrage traite de la fondation de la Section d'études françaises de l'université d'Athènes dans les années 1950. Il est à juste titre signalé que le fait que cette formation devienne universitaire marque un tournant important et le début d'une nouvelle ère. Prenant en charge cette tâche, l'université grecque venait ainsi supplanter l'Institut français dans son rôle de principal formateur des professeurs de français. Mais le nouveau projet, même s'il mettait de côté l'établissement français, brisant du coup le monopole dont ce dernier avait joui depuis les années 1930, s'inscrivait toujours dans le cadre d'une collaboration franco-hellénique. La Section d'études françaises, intégrée à la Faculté des Lettres d'Athènes, assura une formation, dans l'ensemble plus spécialisée, privilégiant les nouvelles approches et méthodes développées dans le domaine $d u$ français langue étrangère. En ce sens, la disciplinarisation des lettres françaises à l'université d'Athènes a marqué un incontestable progrès. Poursuivant son examen du profil des diplômé/es, l'auteure 
procède à des observations fort intéressantes, aidée en cela par les entretiens qu'elle a menés avec des anciennes élèves de la Section.

7 Les recrues étaient, à une écrasante majorité de sexe féminin, accentuant ainsi le phénomène de "surféminisation » déjà observé pendant la période de l'entre-deuxguerres. Presque toutes de nationalité grecque, souvent des anciens ou des anciennes élèves de l'Institut français d'Athènes, elles provenaient majoritairement des couches moyennes et inférieures de la société grecque et des milieux urbains et étaient souvent issues de familles de culture française. Leur origine sociale est intimement liée, selon l'auteure, au choix de la filière universitaire menant au professorat de français: en effet, ce choix fut surtout commandé par des stratégies familiales privilégiant la sécurité professionnelle et la promotion sociale que la carrière professorale assurait. Loukia Efthymiou met également en relief l'opposition qui existait, durant les premières décennies de fonctionnement de la Section française, entre une population étudiante féminine et d'origine grecque et les professeurs Français de sexe masculin qui les formaient. Portant ainsi son regard également sur les «formateurs", elle observe qu'au fil du temps le personnel enseignant de la Section s'est à son tour féminisé et hellénisé. On est ainsi passé du «temps des hommes" au "temps des femmes » et l'hétérogénéité qui caractérisait encore le métier d'enseignant du français à la fin du XIX siècle s'est atténuée un siècle plus tard.

8 L'ouvrage contient une masse impressionnante d'informations que l'auteure s'applique avec succès à rendre intelligibles, en les intégrant dans des schémas interprétatifs convaincants. On peut, certes, regretter que la fondation, dans les années 1950, d'une Section d'études françaises à l'université d'Athènes n'ait pas été suffisamment prise en compte, alors que l'étude, intitulée La formation des francisants en Grèce, ambitionne de traiter de la question à un niveau national. On peut aussi observer que peu de sources du xIX siècle ont été consultées, alors que la période historique étudiée s'étend sur deux siècles. Mais ces observations critiques ne diminuent en rien la validité des résultats de la recherche, ni le mérite d'un travail historique qui, à plusieurs titres, reste exemplaire.

\section{AUTEUR}

NICOLAS MANITAKIS

Université d'Athènes 